\title{
Skipped Zonal based Binary DCT (SZBinDCT) for Image communication over Resource Constrained Visual Sensor Network
}

\author{
G. Suseela*, Asnath Victy Phamila Y \\ School of Computing Science and Engineering, VIT University, Chennai Campus, Chennai-600127, \\ Tamilnadu, India \\ ${ }^{*}$ Corresponding author, e-mail: suseela.g2014phd1186@vit.ac.in
}

\begin{abstract}
The contemporary advancements in CMOS technology and Multimedia systems enabled image communication over resource constrained Visual Sensor Network (VSN). However, the size of image data is huge. It is wise to send the compressed image over the bandwidth limited network. Compression algorithms also should be of energy efficient with low complexity and should offer acceptable image quality. In this work an image coder offering low bit rate less than $0.5 \mathrm{bpp}$ is aimed. In this paper, a low memory and energy efficient skipped zonal based Binary DCT is proposed that codes the transformed samples using Golomb Rice code. Simulation results offered low bitrate 0.39 bpp with PSNR of $27.34 \mathrm{~dB}$ for the standard gray scale test image of Lena size 512 x512 pixels.
\end{abstract}

Keywords: Binary DCT, WSN, Zonal DCT, Image compression, Golomb Rice code

Copyright $@ 2017$ Institute of Advanced Engineering and Science. All rights reserved.

\section{Introduction}

The modern developments in CMOS technology, micro electro mechanical systems (MEMS) and multimedia technology transformed the traditional scalar Wireless Sensor Network (WSN) in to Visual Sensor Network for sensing and communicating visual data as images. Hence, VSN has fostered two dimensional signal processing techniques. The nodes equipped with camera are called as VS nodes. Every VS node has the competency to capture, process and forward the visual data to the sink node. The VSN has long list of applications as like WSN, to name few under the list are surveillance, animal tracking, habitat monitoring, elderly assistance systems, ubiquitous computing and critical infrastructure protection. However, there are certain limitations that are to be considered while designing algorithms for VSN. It is resource limited in terms of processor speed, communication bandwidth, power and memory. Figure 1 depicts the typical architecture of VSN.

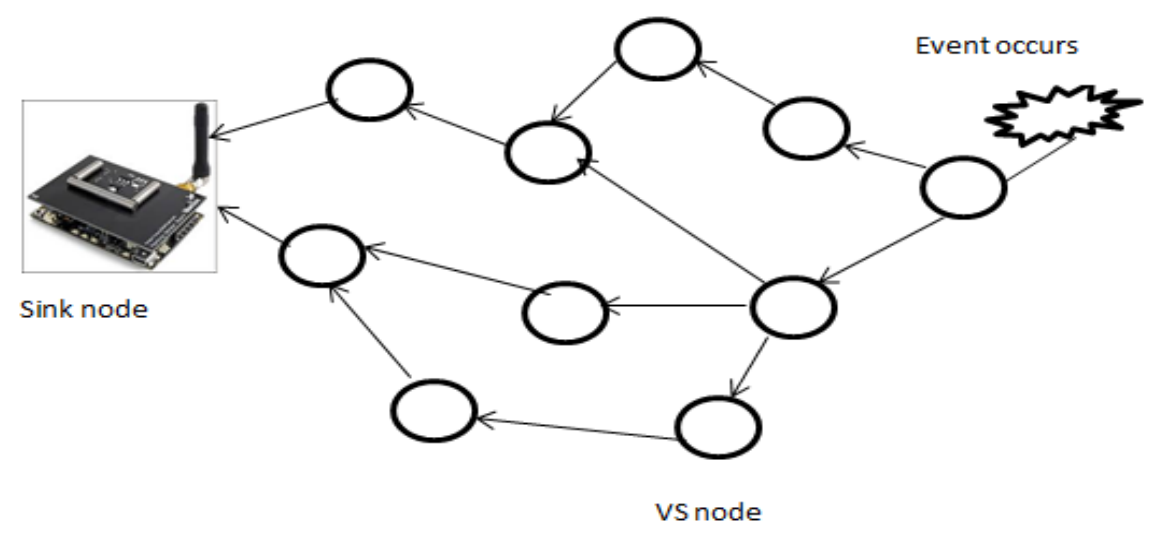

Figure 1. VSN Architecture 
Hence careful adjustments must be carried out for adopting the existing 2-Dimensional Signal processing techniques into VSN. Since 2D image data size is huge, the energy hungry VSN will consume more energy to transmit image, which will drastically reduce the life time of the battery powered wireless network. Hence it is wise to compress the image before transmission using energy efficient image compression algorithms. The standard bench mark algorithms such as JPEG, JPEG 2000 demand more energy for compression than energy required to transmit an uncompressed image leading to putdown the life time of network [1]. To bridge the gap, as a contribution to the research area a low memory and energy competent image coding is proposed named as Skipped Zonal Based Binary DCT (SZ BinDCT) with GRFS (Golomb - Rice Fundamental Sequence) Coding. The traditional dictionary based Huffman coding, arithmetic coding, list based entropy coding like SPHIT, and EZW are complex and require more memory. For low memory implementation GRFS code is best suggested in the literature because of its low complexity and low memory requirement [2].

The paper is organized as follows. Section II discusses about related work in examined domain. Section III discusses the proposed skipped Zonal Based binary DCT. Section IV discusses about Golomb-Rice (GRFS) entropy image coding. Under Section V performance analysis of the proposed is presented in terms of PSNR, CR, bit rate, memory block size and energy consumption. Section VII concludes the paper.

\section{Related Work}

There is wealth of literature in energy efficient image communication over VSN using DWT based and DCT based algorithms. DWT based algorithms offer high quality compression but computational cost is high with respect to energy and memory [3]. DCT based compression algorithms are best suited for image communication over VSN, because of its low memory implementation by $8 \times 8$ tiling style, energy compaction, and coding gain, all leading to good compression ratio[4]. A pure DCT for 2D $8 \times 8$ block requires 466 additions and 96 multiplications [5] which is very high computational load for a light weight processor in the sensor node. More over the processor are not having the hardware funding to process floating point operations of the DCT coefficients. Integer based multiplier less version of DCT is most preferred for VSN platform. Lot of contributions are there in the literature for low complex and fast DCT implementations. Lee et al had presented a platform targeted JPEG with range analysis. They had done three modes of energy efficient compression from slow, medium and fast [6]. Recently Pham et al used fast multiplierless version of DCT using 14 shifters and 38 adders for 1D 8 point DCT called high quality preserving Cordic based Loffler DCT for their change detection system in VSN $[7,8]$.It requires 256 shift operation and 608 additions for 2D 8 point DCT. This computation cost is comparatively higher than BinDCT-C7 [9]. A low memory implementation of DWT based is FrWF using LMBTC is presented by rein et al [10]. They have done five level of wavelet decomposition and LMBTC allows low memory only for larger block sizes.

\section{Skipped Zonal Based Binary DCT (SZBinDCT)}

The proposed SZBinDCT (Skipped Zonal based Binary DCT) is a low memory and energy efficient image transform aimed exclusively for resource constrained platforms such as micro controller embedded sensor motes in the VSN. The traditional way of image compression is a three step chained process i.e. Transform-Quantize-Encode. To this chain the proposed method introduces one more step i.e., pixel block skipping, transfiguring the compression chain as Skip-Transform-Quantize-Encode. The proposed low complex Image coder model is depicted in Figure 2. As a first step of the compression the alternating pixel rows and columns of the entire image is removed; aimed to reduce the redundancy due to neighbouring pixel correlation. By this step $75 \%$ compression is achieved such that the image size is reduced by half in 2D. As a second step of the chained process energy aware transform is identified. BinDCT is a multiplier free integer based DCT transform proposed by Liang and Trac D in 2001[9] which uses only binary shifters and adders. This multiplier free DCT is hardware friendly without the additional hardware requirement for floating point operations. 


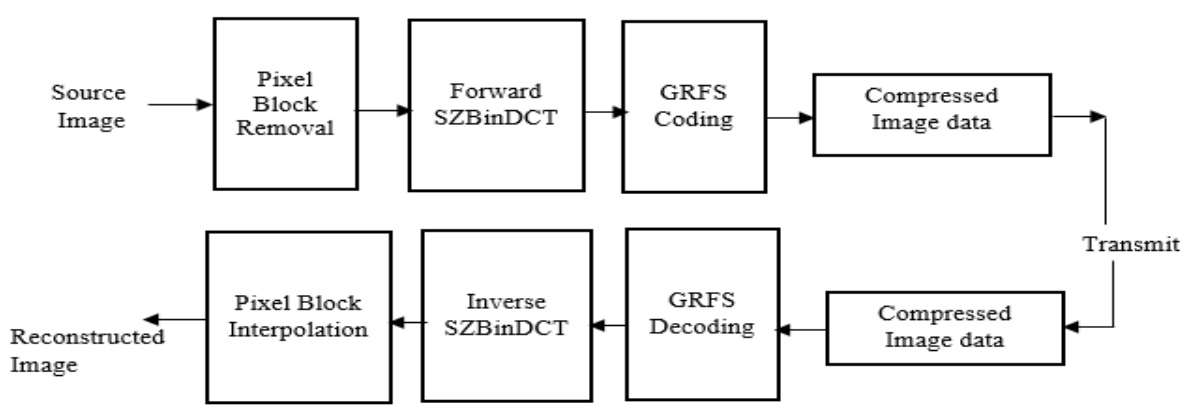

Figure 2.Proposed Image Coder model

The 8 by 8 transform coefficients of Binary DCT (BinDCT) are close approximations of pure 8 by 8 DCT by precomputed dyadic values. BinDCT-C7 requires only as low as 9 shifters and 28 adders for 1D 8 point transform. Therefore the cost of 2D 8 point Binary DCT transform is with 448 additions and 144 shift operations which is very less cost than presented in [8]. BinDCT holds all the properties of DCT like coding gain, energy compaction, and null DC leakage. In the DCT transform matrix the energy points are compacted at top left corner of the matrix. So to have low memory implementation only the top left zone matrix of size $j \times j$ out of the $8 \times 8$ transformed coefficient matrix is considered, computed and encoded in both $1 \mathrm{D}$ and $2 \mathrm{D}$ transforms. For implementation purpose $\mathrm{j}=4$ is chosen, depending upon the requirement on reconstructed image quality and the rate required bit the value of $j$ can be increased or decreased [11]. Only 16 coefficients are encoded out of 64 coefficients. While computing inverse transform at the decoder side all 48 missing coefficients are assumed as zeros as shown in Figure 3. The third step of the compression is quantization. In the proposed system quantization is done using binary shift operation without using floating point operations. The quantized coefficients are coded using GRFS coder.

\begin{tabular}{|llllllll|}
\hline DC & $\mathbf{1 0}$ & $\mathbf{7}$ & $\mathbf{1}$ & 0 & 0 & 0 & 0 \\
$\mathbf{8}$ & $\mathbf{- 2}$ & $\mathbf{3}$ & $\mathbf{4}$ & 0 & 0 & 0 & 0 \\
-4 & $\mathbf{1}$ & $\mathbf{0}$ & $\mathbf{- 1}$ & 0 & 0 & 0 & 0 \\
$\mathbf{3}$ & $\mathbf{1}$ & $\mathbf{0}$ & $\mathbf{3}$ & 0 & 0 & 0 & 0 \\
0 & 0 & 0 & 0 & 0 & 0 & 0 & 0 \\
0 & 0 & 0 & 0 & 0 & 0 & 0 & 0 \\
0 & 0 & 0 & 0 & 0 & 0 & 0 & 0 \\
0 & 0 & 0 & 0 & 0 & 0 & 0 & 0 \\
\hline
\end{tabular}

Figure 3. SZBinDCT $8 \times 8$ transform matrix at Decoder

\section{Golomb Rice Fundamental Sequence (GRFS) Coding}

Golomb Rice code is based on Low complexity and Low memory Entropy Coder (LLEC) implemented in [2]. In this coding two key tasks are carried out. First the transformed coefficients are ordered as a special tree structure called Zero Tree, to exploit parent child relationships among the transformed and quantized coefficient. It is assumed that after quantization of transform coefficients if a coefficient is insignificant (means value $=0$ ), all the coefficients of same orientation in the matrix will be insignificant. All such insignificant zeros are coded using ZTC. Remaining significant coefficients are coded using GRFS code. ZTC works well if in coarser version of sample matrix contains lot of zeros. In the proposed compression technique, only the significant coefficients ( $\mathrm{j}=4$, energy compacted zone) are considered for coding, So ZTC of LLEC is not required. By excluding the ZTC stage of LLEC the complexity is further reduced. GRFS code directly applied to the quantized sample matrix. The powerful technique used in JPEG for bit reduction is coefficient bucketing. That is the quantised 
coefficients are apportioned into different buckets as shown in Table 1. GRFS code works based on coefficient bucketing. For a value $\mathrm{x}$ its bucket number and its position inside the bucket is found. Then $x$ is coded using GRFS coding as follows,

\section{GRFS(x)}

1. $\quad \mathrm{Y}=\operatorname{bucket}(\mathrm{x})$ is identified

2. $\quad \mathrm{p}(\mathrm{x})=$ position of $\mathrm{x}$ inside the $\mathrm{Y}$ is identified

3. $\quad \mathrm{Y}$ is coded with $\mathrm{Y}$ number of zeros followed by 1.

4. $\quad \mathrm{p}(\mathrm{x})$ is coded in binary with $\mathrm{Y}$ bits.

5. Code $=Y+p(x)$, Where ' + ' is concatenation.

Table 1.GRFS coefficient bucketing [2]

\begin{tabular}{lc}
\hline Bucket & Coefficient Values \\
\hline 0 & 0 \\
1 & $-1,1$ \\
2 & $-3,-2,2,3$ \\
3 & $-7,-$ to $-4,4$ to 7 \\
4 & -15 to $-8,8$ to 15 \\
5 & -31 to $-16,16$ to 3 \\
6 & -63 to $-32,32$ to 63 \\
7 & -127 to $-64,64$ to 127 \\
8 & -255 to $-128,128$ to 255 \\
\hline
\end{tabular}

\section{Experimental Results and Analysis}

For implementation purpose, SZBinDCT is experimented with various block sizes for pixel removal such as $1 \times 1,2 \times 2,4 \times 4$ and $8 \times 8$. The PSNR and entropy offered by SZBinDCT for standard gray scale test images[12] Lena, Barbara, house peppers and baboon all of size $512 \times 512$ for $1 \times 1,2 \times 2$ and $4 \times 4$ (pixel removal block sizes) were presented in Table 3 and in Figure 4 . The results were promising for $1 \times 1$ and $2 \times 2$ skipped blo.

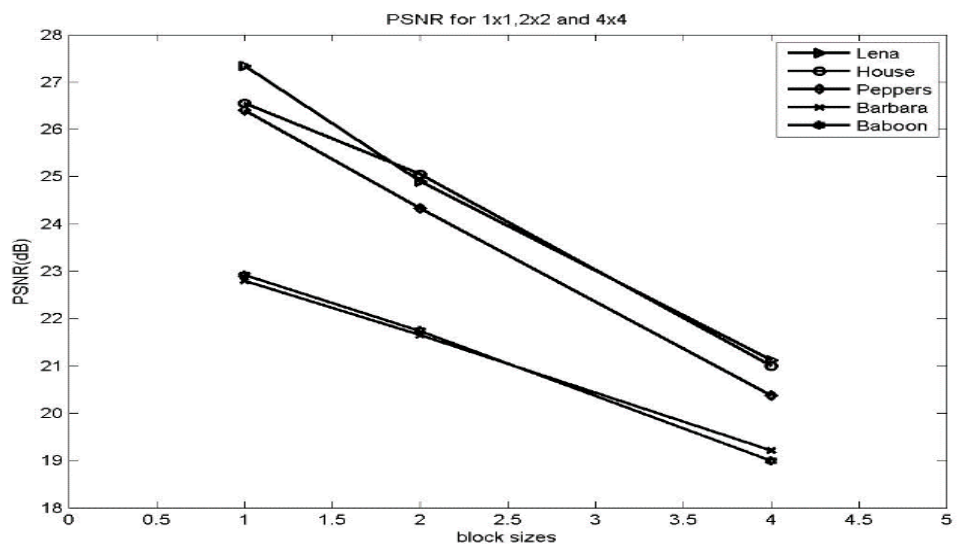

Figure 4 Skipped block size Vs PSNR of Test Images.

\subsection{Energy Analysis}

Simulations were carried out at the target platform using AVR Studio 20060421. As the mica2 mote is built up with Atmel's ATmega128 microcontroller (With operating frequency of 8 $\mathrm{MHz}$, and an active power consumption of $22 \mathrm{~mW}$ ), it is used as the target platform for simulation by setting "-O3" optimization in the compiler. The simulation results were presented under Table 3 for an $8 \times 8$ image block, it infers that the proposed scheme consumes very less energy of $11.352 \mu \mathrm{J}$ and low memory of 4254 bytes. The proposed system outperforms the Independent JPEG Group (float) and Platform oriented JPEG [6] in terms of memory and energy consumption. The proposed SZBinDCT with GRFS requires only $0.48 \%$ of the energy consumed by IJG (float) and $12.64 \%$ of the energy consumed by Lee et al (fast) [6]. Also 
requires only $19.5 \%$ and $23 \%$ of memory used by IJG (float) and Lee et al (fast) respectively. The compression ratio achieved is $81.2 \%$.

Table 2. PSNR, Bitrate of the proposed scheme for $1 \times 1,2 \times 2$ and $4 \times 4$ pixel block removal

\begin{tabular}{ccccccc}
\hline Image & \multicolumn{6}{c}{ Pixel Removal block size } \\
& $\begin{array}{c}\text { PSNR } \\
\text { (dB) }\end{array}$ & $\begin{array}{c}\text { Bitrate } \\
(\mathrm{bpp})\end{array}$ & $\begin{array}{c}\text { PSNR } \\
\text { 2x2 }\end{array}$ & $\begin{array}{c}\text { Bitrate } \\
(\mathrm{dBp})\end{array}$ & $\begin{array}{c}\text { PSNR } \\
(\mathrm{dB})\end{array}$ & $\begin{array}{c}\text { Bitrate } \\
(\mathrm{bpp})\end{array}$ \\
\hline Lena & 27.34 & 0.39 & 24.90 & 0.40 & 21.12 & 0.37 \\
Barbara & 22.80 & 0.43 & 21.65 & 0.44 & 20.37 & 0.42 \\
House & 26.55 & 0.41 & 25.05 & 0.42 & 21.76 & 0.40 \\
Peppers & 26.40 & 0.41 & 24.33 & 0.41 & 20.37 & 0.38 \\
Baboon & 22.92 & 0.49 & 21.74 & 0.50 & 18.96 & 0.49 \\
\hline
\end{tabular}

Table 3. Energy Consumption for an 8x8 image block in target platform Atmel ATmega 128

\begin{tabular}{lllllll}
\hline Scheme & $\begin{array}{l}\text { Transform } \\
\text { cycles }\end{array}$ & $\begin{array}{l}\text { Quantization+ } \\
\text { Coding cycles }\end{array}$ & $\begin{array}{l}\text { Total } \\
\text { Cycles }\end{array}$ & $\begin{array}{l}\text { Memory } \\
\text { block } \\
\text { (bytes) }\end{array}$ & $\begin{array}{l}\text { Execution } \\
\text { time } \\
(\mathrm{ms})\end{array}$ & $\begin{array}{l}\text { Energy } \\
(\mu \mathrm{J})\end{array}$ \\
\hline JPEG(float) & 580106 & 266344 & 846450 & 21806 & 105.80 & 2327.74 \\
Lee et al(fast) & 8678 & 23889 & 32657 & 18504 & 4.08 & 89.81 \\
SZBinDCT+GRFS & 3135 & 993 & 4128 & 4254 & 0.516 & 11.352 \\
\hline
\end{tabular}

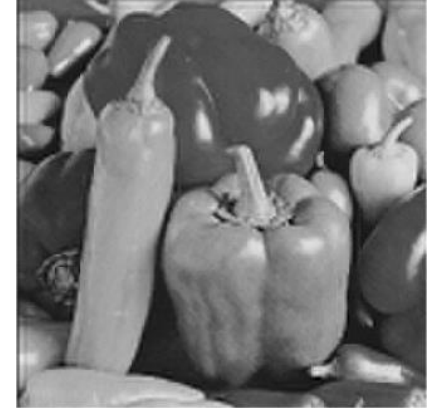

PSNR $=26.40 \mathrm{~dB}$ bitrate $=0.41$

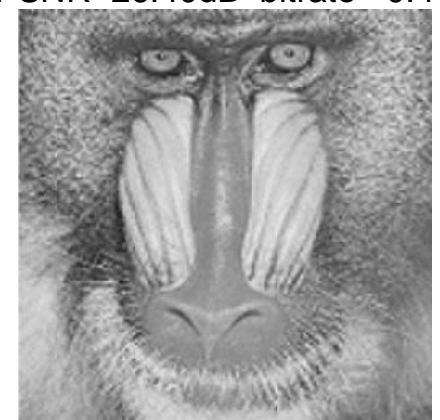

PSNR $=22.92 \mathrm{~dB}$ bitrate $=0.49$

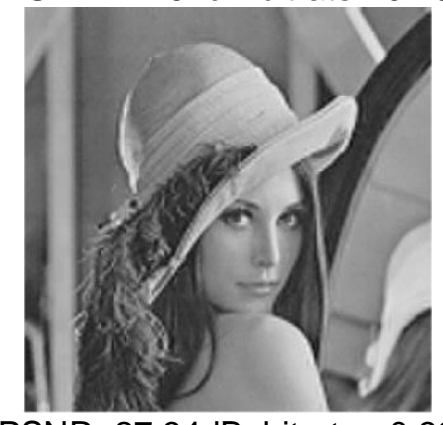

PSNR $=27.34 \mathrm{~dB}$ bitrate $=0.39$

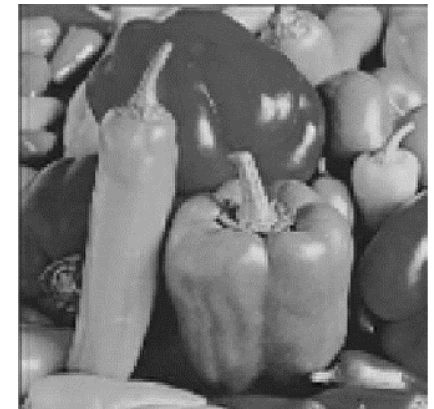

$\mathrm{PSNR}=24.33 \mathrm{~dB}$ bitrate $=0.41$

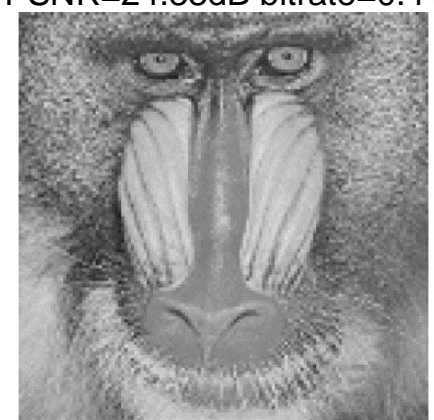

PSNR $=21.73 \mathrm{~dB}$ bitrate $=0.50$

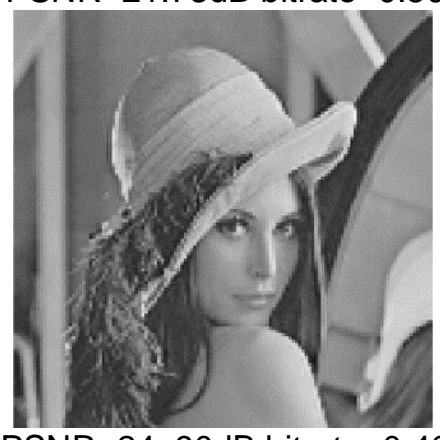

PSNR $=24 . .90 \mathrm{~dB}$ bitrate $=0.40$ 


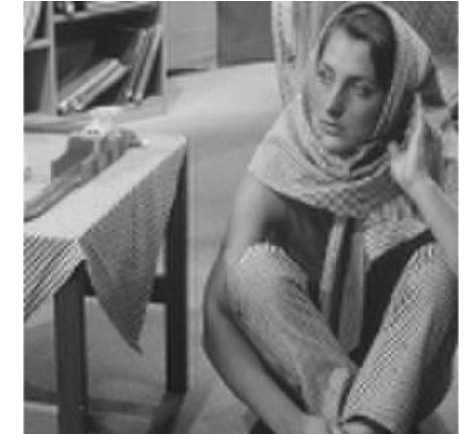

PSNR $=22.80 \mathrm{~dB}$ bitrate $=0.43$

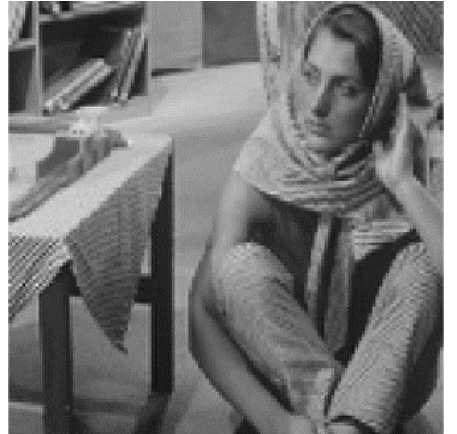

PSNR $=21.65 \mathrm{~dB}$ bitrate $=0.44$

\section{CONCLUSION}

In this paper an energy efficient and low memory SZBinDCT with GRFS image coder for image communication in VSN is presented . The image coder ensures energy efficient image compression, involving low computational load and low memory; hence consumes less energy. SZBinDCT computes only $4 \times 4$ transform coefficients and codes using GRFS code without sorting passes involved in ZTC, thus consuming less memory. Simulation results shown that under target platform Atmel ATmega128 processor requires only $11.2 \mu \mathrm{J}$ for coding (transform + quantization +entropy coding) an 8x8 block. Considerable energy savings have been achieved by the compression algorithm; Transmission energy will also be reduced because of the low bit rate offered by the proposed system hence SZBinDCT with GRFS image coder will promise extended life period of low power resource constrained VSN.

\section{References}

[1] M. L. Kaddachi, A. Soudani, V. Lecuire, K. Torki, L. Makkaoui, and J.-M. Moureaux, "Low power hardware-based image compression solution for wireless camera sensor networks," Computer Standards \& Interfaces, vol. 34, no. 1, pp. 14-23, Jan. 2012.

[2] Debin Zhao, Y. K. Chan, and Wen Gao, "Low Complexity and low memory Entropy Coder for Image Compression ",IEEE Transactions on Circuits and Systems for Video Technology, Vol.11, no.10, pp.1140-1145,2001

[3] Y. A. V. Phamila and R. Amutha, "Low-complex energy-aware image communication in visual sensor networks," Journal of Electronic Imaging, vol. 22, no. 4, p. 041107, Aug. 2013.

[4] Trac D. Tran,"The BinDCT:Fast Multiplierless Approximations of the DCT", IEEE Signal Processing Letters, Vol 7, No 6, June 2000

[5] N. I. Cho and S. U. Lee, "Fast algorithm and implementation of 2-D discrete cosine transform," IEEE Transactions on Circuits and Systems, vol. 38, no. 3, pp. 297-305, Mar. 1991.

[6] Lee, D.U., Kim, H., Rahimi, M., Estrin, D. and Villasenor, J.D., 2009. Energy-efficient image compression for resource-constrained platforms. IEEE Transactions on Image Processing, 18(9), pp.2100-2113.

[7] Pham, Congduc, and Vincent Lecuire. "Building low-cost wireless image sensor networks: from single camera to multi-camera system." In Proceedings of the 9th International Conference on Distributed Smart Cameras, pp. 158-163. ACM, 2015.

[8] Heyne, Benjamin, Chi-Chia Sun, Juergen Goetze, and Shanq-Jang Ruan. "A computationally efficient high-quality cordic based DCT." In Signal Processing Conference, 2006 14th European, pp. 1-5. IEEE, 2006.

[9] Liang and Trac D. Tran, "Fast Multiplierless Approximations of the DCT With the Lifting Scheme", IEEE Transactions on Signal Procesing, Vol. 49, No. 12, December 2001.

[10] M. Tausif, N. R. Kidwai, E. Khan, and M. Reisslein, "FrWF-Based LMBTC: Memory-efficient image coding for visual sensors," IEEE Sensors Journal, vol. 15, no. 11, pp. 6218-6228, Nov.2015

[11] V. Lecuire, L. Makkaoui, and J. Moureaux, "Fast zonal DCT for energy conservation in wireless image sensor networks," Electronics Letters, vol. 48, no. 2, p. 125, 2012.

[12] J.Kominek, Waterloo BragZone University of Waterloo, http://links.uwaterloo.ca/Repository.html (7 August 2015). 